\title{
BMJ Open Can socioeconomic health differences be explained by physical activity at work and during leisure time? Rationale and protocol of the active worker individual participant meta-analysis
}

\author{
Pieter Coenen, ${ }^{1}$ Maaike A Huysmans, ${ }^{1}$ Andreas Holtermann, ${ }^{2}$ Richard Troiano, ${ }^{3}$ \\ Paul Jarle Mork, ${ }^{4}$ Steinar Krokstad, ${ }^{4}$ Els Clays, ${ }^{5}$ Willem van Mechelen, ${ }^{1}$ \\ Allard J van der Beek ${ }^{1}$
}

\section{To cite: Coenen $P$,}

Huysmans MA, Holtermann A, et al. Can socioeconomic health differences be explained by physical activity at work and during leisure time? Rationale and protocol of the active worker individual participant meta-analysis. BMJ Open 2018;8:e23379. doi:10.1136/ bmjopen-2018-023379

\section{- Prepublication history and} additional material for this paper are available online. To view these files, please visit the journal online (http://dx.doi org/10.1136/bmjopen-2018023379).

Received 13 April 2018 Revised 7 August 2018 Accepted 25 September 2018

Check for updates

(C) Author(s) (or their employer(s)) 2018. Re-use permitted under CC BY-NC. No commercial re-use. See rights and permissions. Published by BMJ.

For numbered affiliations see end of article.

Correspondence to Dr Pieter Coenen; p.coenen@vumc.nl

\section{ABSTRACT}

Introduction Socioeconomic health differences have often been described, but remain insufficiently understood. Recent evidence suggests that workers who are high (compared with low) physically active at work are less healthy. Moreover, workers who are highly physically active at work are predominantly physically inactive during leisure time. These observations suggest that workers with a lower socioeconomic status may be exposed to negative health consequences of occupational physical activity and may only benefit to a limited extent from health benefits of leisure-time physical activity. Physical activity may therefore be an important driver of socioeconomic health differences. We describe the rationale and protocol of the active worker study, an individual participant data meta-analysis aimed at exploring socioeconomic health differences by differential doses of physical activity at work and leisure time.

Methods and analysis Using database and scoping searches (we searched in PubMed, Embase, CINAHL, PsycINF0 and Evidence-Based Medicine Reviews from database inception to 14 September 2017), we have identified 49 published and unpublished prospective studies in which the association of occupational and leisure-time physical activity with cardiovascular or allcause mortality was assessed. Principal investigators of these studies will be invited to participate in the active worker consortium, after which data will be retrieved. After data merging and harmonising, we will perform multilevel survival analysis assessing the combined association of occupational and leisure-time physical activity with mortality. We will also test the mediating effect of physical activity on the association of socioeconomic status and mortality (ie, socioeconomic health differences).

Discussion The Medical Ethical Committee of the VU University Medical Center has declared, according to Dutch legislation, that the 'Dutch Medical Research Involving Human Subjects Act' does not apply to the current study. As such, no ethics approval is required. We intent to publish outcomes of the active worker Study in scientific peer-reviewed journals.

PROSPERO registration number CRD42018085228.
Strengths and limitations of this study

- The current individual participant data (IPD) meta-analysis of the active worker study consortium will explore the differential effects of occupational and leisure-time physical activity on cardiovascular and all-cause mortality.

- Apart from our already completed search for eligible studies and thorough analysis and data management plan, the strength of our IPD meta-analysis lies in the ability to detect possible relevant interaction and mediation effects.

- This will allow us to study the role of occupational and leisure-time physical activity in socioeconomic health differences, while also exploring relevant subgroups (eg, with pre-existing conditions).

- A potential limitation of our study is the possibility of not being able to obtain data from all studies that have been identified.

- Another potential limitation is the heterogeneity in study data, possibly limiting the ability to harmonise data.

\section{BACKGROUND}

People with a lower socioeconomic status have an increased risk of premature cardiovascular and all-cause mortality, compared with those with a higher socioeconomic status. ${ }^{12}$ To maintain a sustainable workforce, a better understanding of socioeconomic health differences, in particular among the working population, is required. Socioeconomic health differences have often been described, and have been found to be associated with a range of demographic (eg, age, ethnicity and marital status), behavioural (eg, physical activity, smoking, alcohol use and diet), psychosocial (eg, stress, social support and psychosocial work demands) and material factors (eg, healthcare utilisation and 
neighbourhood conditions)..$^{3-6}$ However, socioeconomic health differences remain poorly understood, as they cannot be fully attributed to these factors. A recent systematic review showed that psychosocial factors explain $4 \%-49 \%$, behavioural factors $7 \%-45 \%$, while material factors explain $11 \%-76 \%$ of health differences. ${ }^{7}$

To date, physical activity is considered an important and significant behaviour for the prevention of premature death from many lifestyle-related diseases. ${ }^{8}$ The health implications of engagement in different domains of physical activity (according to common PA research: leisure time, work, household and transport ${ }^{9}$ ) are considered to be positive and alike. Workers, who are physically active at work, most of them with low socioeconomic status, could therefore be expected to be healthier and live longer. Surprisingly, however, recent evidence suggests that workers who are highly physically active at work are less healthy and die earlier than those who are less physically active at work. ${ }^{10}{ }^{11}$ This evidence shows a consistently reduced risk of lifestyle-related diseases associated with high doses of leisure-time physical activity, but an increased risk of cardiovascular diseases ${ }^{12-15}$ and mortality ${ }^{16-18}$ with high doses of occupational physical activity (even when adjusted for current health status, other lifestyle factors and socioeconomic status), suggesting a physical activity paradox. ${ }^{19}$ We have recently found evidence for this paradox in a systematic review, showing an increased risk of premature mortality among men who are highly physically active at work, compared with those who engage in low doses of occupational physical activity (HR; with 95\% CI 1.18 (1.05 to 1.34$))^{.0}$

Moreover, workers from lower socioeconomic status are often physically inactive during their leisure time, ${ }^{21}$ as these workers spend most of their daily physical activity time at work. ${ }^{22}$ The above suggests that workers with a lower socioeconomic status may be exposed to the negative health consequences of occupational physical activity and may only benefit to a limited extent from the positive health consequences of leisure-time physical activity. As such, the differential effects of physical activity at work and leisure time may be an important driver of socioeconomic health differences. $^{23}$

To improve the health of workers with a lower socioeconomic status and to maintain a sustainable workforce, more knowledge on the opposing health effects of occupational and leisure-time physical activity is urgently required. Presently, a knowledge gap exists as to what type of interventions may work for whom and when. It is therefore important to identify workers who may benefit the most from physical activity interventions, to indicate what elements such interventions should consist of, and to identify barriers and facilitators of certain interventions. Such knowledge can inform physical activity-based interventions and public health guidelines, thus contributing to a reduction of socioeconomic health differences. An individual participant data (IPD) meta-analysis is essential to fill the knowledge gap indicated above. In contrast to a study-level meta-analysis, an IPD meta-analysis also allows for testing of interaction, mediation or moderation. ${ }^{24}$ This allows for the possibility of identifying relevant subgroups and the ability to test mechanistic pathways. ${ }^{25}{ }^{26}$ Moreover, an IPD meta-analysis has the benefit of a larger number of data points, facilitating more statistically powerful and sound conclusions based on careful evaluation of modelling assumptions. An IPD meta-analysis also allows us to account for missing data at the individual participant level and to standardise analytical techniques, inclusion criteria and outcome definitions across studies.

In this manuscript, we describe the rationale and protocol of the active worker study, an IPD meta-analysis carried out by the active worker consortium. The primary aim of this study is to explore socioeconomic health differences related to physical activity at work and during leisure time. To do so, we will:

1. Assess the combined effect of occupational and leisure-time physical activity on cardiovascular and allcause mortality.

2. Identify subgroups (eg, with pre-existing hypertension or obesity) that are particularly vulnerable for this effect.

3. Identify subgroups (eg, with high levels of cardiorespiratory fitness) that have a differential association between occupational and leisure-time physical activity and cardiovascular and all-cause mortality.

4. Assess the independent contribution of occupational and leisure-time physical activity to socioeconomic health differences.

Figure 1 presents a comprehensive conceptual model for the hypothesised pathway of the association of socioeconomic status with health outcomes in which also relevant other factors (including demographic, behavioural, psychosocial and material factors) and pre-existing conditions are considered. Socioeconomic status is hypothesised to be associated with physical activity, with more occupational physical activity and less leisure-time physical activity among those with a low socioeconomic status and vice versa. Physical activity (which is inter-related with a range of relevant other factors, including demographic, behavioural, psychosocial and material factors and pre-existing conditions) is subsequently associated with health outcomes, such as cardiovascular and all-cause mortality. As such, it is hypothesised that physical activity (at work and during leisure time) plays an important mediating/ moderating role in socioeconomic health differences.

\section{METHODS/DESIGN}

The current manuscript is prepared in accordance with thePreferred Reporting Items for Systematic Reviews and Meta-Analyses Protocols (PRISM) statement. ${ }^{27}$ The described IPD meta-analysis has been a priori registered in PROSPERO.$^{28}$ Conducting and reporting of the proposed IPD meta-analysis will be done in accordance with methods described by the Cochrane IPD Meta-analysis Methods Group ${ }^{25}$ and the PRISMA-IPD statement. ${ }^{29}$ Methodologies for this IPD meta-analysis were based on previous work. ${ }^{30} 31$ 


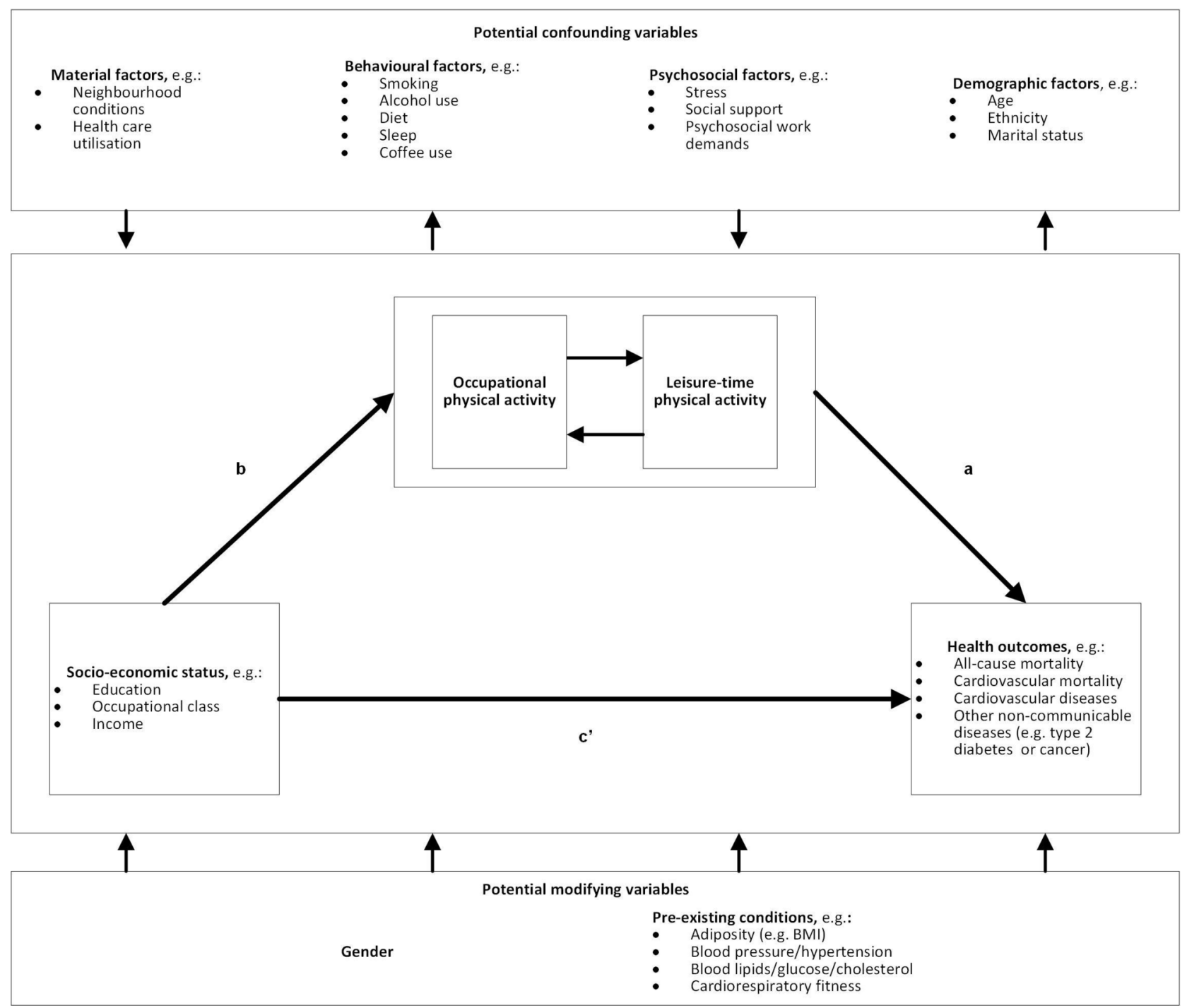

Figure 1 Conceptual model for the pathway of the combined role of occupational and leisure-time physical activity in socioeconomic health differences. Socioeconomic status is hypothesised to be associated with physical activity, with more leisure-time physical activity and less occupational physical activity among those with a high socioeconomic status and vice versa. Physical activity (which is inter-related with a range of relevant other factors, including demographic, behavioural, psychosocial and material factors as well as pre-existing conditions) is subsequently associated with health outcomes, hence resulting in socioeconomic health differences. To address aim 1, we will assess the combined association of occupational and leisure-time physical activity with the primary outcome (pathway a). For aims 2 and 3 , we will additionally consider potential modifying effects. To address research aim 4, we will study the mediating effects of occupational and leisure-time physical activity on the association of socioeconomic status and the primary outcome. To do so, we will provide an estimate of the relative strength of the mediation effect $(\mathrm{axb})$ and we will be comparing this to the total direct effect (c'), using the product-ofcoefficients test. In all these models, potential confounders will be considered. BMI, body mass index.

\section{The consortium, data sources and study selection}

Several strategies have been used to identify published and unpublished eligible studies, including literature searches in electronic databases and scoping searches through personal communication with experts in the field, collaborators and colleagues. The literature was searched systematically in the bibliographic databases of PubMed, Embase, CINAHL, PsycINFO and Evidence-Based Medicine Reviews from database inception to 14 September 2017. Search terms included terms expressing physical activity and mortality (see online supplementary material 1 for more details). We searched for original prospective studies, in which the association of both occupational and leisure-time physical activity on cardiovascular or all-cause mortality in adult workers (ie, 18-65 years of age at the time of physical activity assessment) was assessed, taking workers' socioeconomic status (ie, expressed as education, occupational class and/or income) into account. Studies assessing physical activity at the level of the individual were included. Studies were excluded that had assessed occupational physical activity based on task and/ or job classification (eg, a binary distinction of blue-collar vs white-collar workers or manual vs non-manual work). Only studies in which cardiovascular or all-cause mortality 
was expressed as a time-to-event variable (ie, occurrence of the event-yes/no, and the time to the possible event) were included to allow for the execution of survival analysis.

The literature search generated 2490 unique references that were screened on their title and/or abstract.
A total of 174 full-text articles were retrieved of which 76 were excluded for various reasons (online supplementary material 2). After adding 35 articles from other sources, 49 unique studies (from 106 articles) were identified meeting our inclusion criteria for being eligible to participate in the active worker consortium (figure 2;

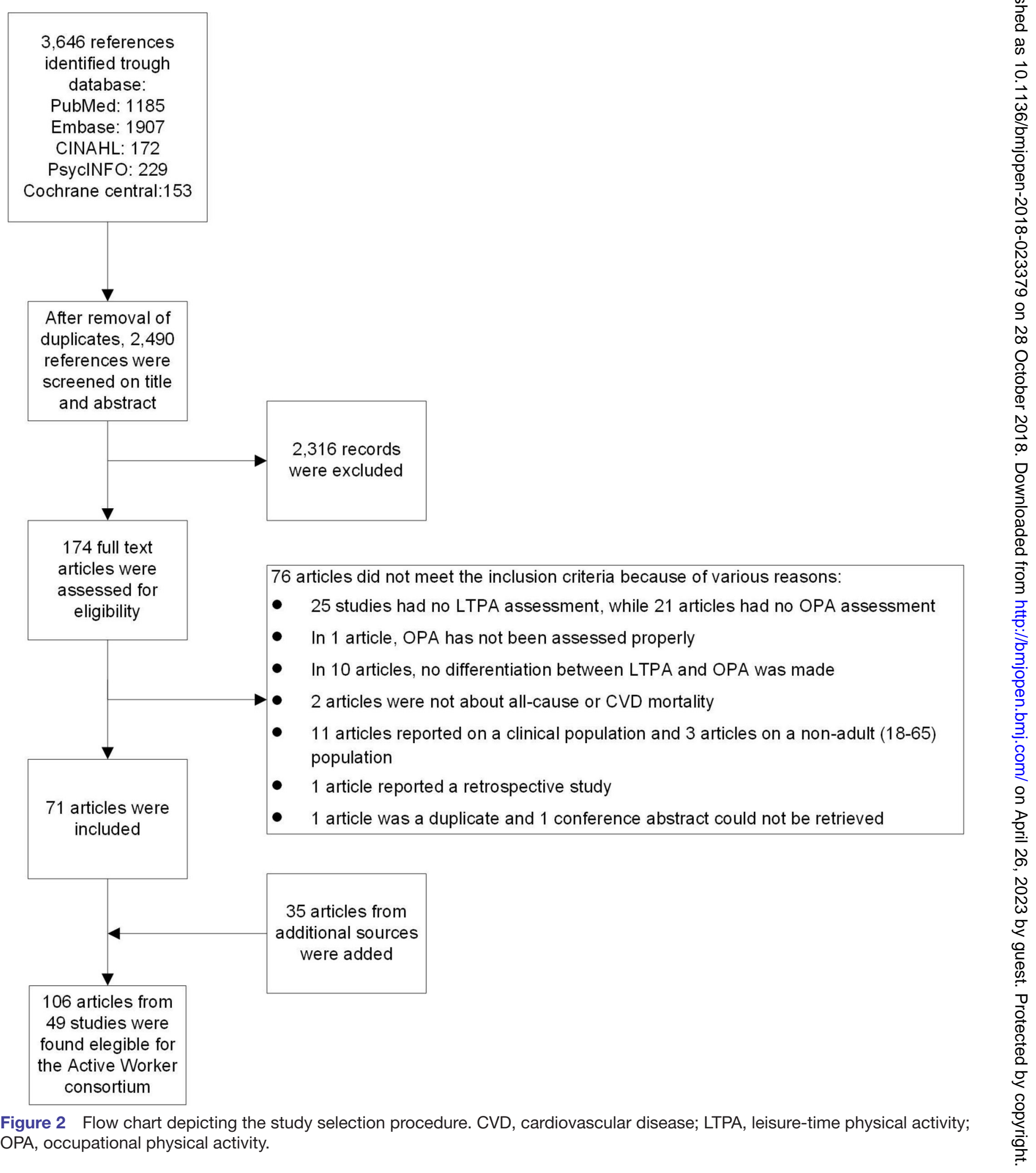


online supplementary material 3). From these eligible studies, corresponding authors, principal investigators and/or researchers from the network of the consortium members will be invited to collaborate in our active worker study consortium. Invitations will be sent by email. After no response, a reminder email will be sent and/or a telephone call will be made to achieve participation. Participation in the consortium includes sharing of data and ideas, and manuscript preparation. Reasons for refusal to participate will be noted.

After agreeing to collaborate, potential collaborators will be asked to fill out our data request form in order for us to obtain more information on the respective cohorts (eg, regarding contact details of principal investigators, study design and available data). Also, researchers will be asked to sign a policy document after which they will be asked to transfer their (anonymised and encrypted) data.

\section{Data acquisition and harmonisation}

Data on occupational and leisure-time physical activity (predictor variables; typically not including the domains of transport and household), all-cause mortality (primary outcomes) or cardiovascular diseases or mortality (secondary outcomes), socioeconomic status and relevant other factors (including demographic, behavioural, psychosocial and material factors and pre-existing conditions) will be retrieved from each of the participating studies (as described in more detail in table 1 and figure 1). Study data with at least the predictor variables, primary outcome variables and socioeconomic status will be used for further analysis. For each of the variables, the most detailed level of data (ie, continuous variables rather than categorical ones) will be requested.

Collaborators will be asked to supply raw data that can be transferred in any electronic format (eg, SPSS, SAS, Stata or Excel) in an anonymised file (ie, only labelling participants by a unique identification number). Data of all studies will be harmonised by labelling and recoding variables and items. Of continuous variables, Z-scores will be calculated to be able to harmonise constructs measured by different questionnaires/items. The most detailed level of data possible will be used for data harmonisation (eg, continuous variables rather than categorical ones). However, if needed categorical variables from different studies will be harmonised, classifying them into predetermined categories.

For physical activity variables (both occupational and leisure time), we intent to express all data into MET-hours / week, using established scoring systems, such as for the International physical activity questionnaire (IPAQ) ${ }^{32}$ and for the US Census Occupational Classification System, ${ }^{33}$ and the Compendium of Physical Activities. ${ }^{34}$ If available, such continuous data can be harmonised across studies and modelled as such, according to the below-mentioned analysis plan. In an additional sensitivity analysis, also considering studies without continuous MET-based data, physical activity data will be categorised into the four categories from the physical activity continuum (ie, sedentary
Table 1 Overview of variables that will be requested from eligible studies

\begin{tabular}{|c|c|c|}
\hline Variable group & Variables & Relevant details \\
\hline Predicting variables & $\begin{array}{l}\text { Occupational } \\
\text { physical activity } \\
\text { Leisure-time } \\
\text { physical activity }\end{array}$ & $\begin{array}{l}\text { Self-reports. If possible, } \\
\text { frequency, duration } \\
\text { and intensity of } \\
\text { physical activity will be } \\
\text { considered. }\end{array}$ \\
\hline Socioeconomic status & $\begin{array}{l}\text { Education level } \\
\text { Occupational class } \\
\text { Income }\end{array}$ & \\
\hline Primary outcomes & All-cause mortality & $\begin{array}{l}\text { Time to occurrence, } \\
\text { ascertained by national } \\
\text { or local registers. }\end{array}$ \\
\hline Secondary outcomes & $\begin{array}{l}\text { Cardiovascular } \\
\text { mortality } \\
\text { Cardiovascular } \\
\text { diseases in general } \\
\text { Specific } \\
\text { cardiovascular } \\
\text { diseases (eg, } \\
\text { ischaemic heart } \\
\text { disease, myocardial } \\
\text { infarction or stroke) }\end{array}$ & $\begin{array}{l}\text { Time to occurrence, } \\
\text { ascertained by national } \\
\text { or local registers or self- } \\
\text { reports. }\end{array}$ \\
\hline Pre-existing conditions & $\begin{array}{l}\text { Adiposity (eg, body } \\
\text { mass index, waist } \\
\text { circumference or fat } \\
\text { percentage) } \\
\text { Blood pressure and/ } \\
\text { or hypertension } \\
\text { Blood lipids, } \\
\text { cholesterol and/or } \\
\text { glucose } \\
\text { Cardiorespiratory } \\
\text { fitness } \\
\text { Self-reported health } \\
\text { Other non- } \\
\text { communicable } \\
\text { diseases (eg, } \\
\text { diabetes, cancer, } \\
\text { pulmonary diseases, } \\
\text { mental health } \\
\text { condition) }\end{array}$ & \\
\hline \multicolumn{3}{|l|}{ Other relevant factors } \\
\hline Demographic factors & $\begin{array}{l}\text { Gender } \\
\text { Age } \\
\text { Ethnicity } \\
\text { Marital status }\end{array}$ & \\
\hline Behavioural factors & $\begin{array}{l}\text { Smoking } \\
\text { Alcohol use } \\
\text { Diet (eg, } \\
\text { consumption of fruit } \\
\text { and vegetables, } \\
\text { sweets, snacks and } \\
\text { sugary drinks) } \\
\text { Sleep } \\
\text { Coffee use } \\
\text { Other (eg, transport } \\
\text { and/or household) } \\
\text { domains of physical } \\
\text { activity }\end{array}$ & \\
\hline Psychosocial factors & $\begin{array}{l}\text { Stress } \\
\text { Social support } \\
\text { Psychosocial work } \\
\text { demands (eg, } \\
\text { decision authority, } \\
\text { effort-reward } \\
\text { imbalance, job } \\
\text { strain) }\end{array}$ & \\
\hline Material factors & $\begin{array}{l}\text { Healthcare } \\
\text { utilisation } \\
\text { Medication use } \\
\text { Neighbourhood } \\
\text { conditions }\end{array}$ & \\
\hline
\end{tabular}


behaviour, low, moderate and high), using established cut-off values. ${ }^{35}$ The latter will be done during a consensus meeting with the active worker study core group.

Once the original data file has been received and harmonised, data will be transferred to a single file by the help of the VU University Medical Center data management department. All data will be checked for consistency, which includes identifying potential outliers and missing data, after which queries will be discussed and resolved directly with the responsible collaborator.

\section{Risk of bias assessment}

Risk of bias assessment of each of the studies will be performed by two reviewers independently, using a published Cochrane scoring system, ${ }^{36}$ with six criteria related to study participation, attrition, predictor variable measurements and outcomes (online supplementary material 4 ). Summary scores will be calculated and expressed on a $0 \%-100 \%$ scale; summary scores $>75 \%$ considered to be of high methodological quality, hence, low risk of bias. In a separate analysis, we will assess the possible role of risk of bias on our findings.

\section{Data analysis}

Included studies will be described according to their data and risk of bias. Meta-analyses will be performed using multilevel (with the different studies being the different levels) survival regression analysis with SPSS (V.22). Review Manager (RevMan, the Cochrane Collaboration) will be used for pooling of individual study data and for plotting of the data. In forest plots, HRs will be reported with $95 \%$ CI depicting each individual study, as well as pooled associations of occupational and leisure-time physical activity with the primary outcome. In all analyses, men and women will be considered separately anyway. Heterogeneity among studies will be assessed using $\mathrm{I}^{2}$ statistics and visual inspection of the forest plots will be performed, while subgroup analyses will be conducted using $\chi^{2}$ statistics (testing for the effect of risk of bias, way of assessment of physical activity and heterogeneity of the study sample). A sensitivity analysis will be performed to assess the effect of individual study findings on the pooled results. Funnel plots will be generated to assess publication bias (through visual inspection).

A model outlining the analyses is shown in figure 1 . To address aims 1-3, multivariate survival regression will be conducted, testing for the combined association of occupational and leisure-time physical activity with the primary outcome (pathway a in figure 1). For aim 1, we will test the combined association of occupational and leisure-time physical activity. For aims 2 and 3, additional interaction terms will be included, providing information regarding potential effect modifying effects. This interaction term will gain insight into whether or not the effect of occupational and leisure-time physical activity on the outcome differs across different modifier categories. Here, potential effect modifying factors such as chronic lifestyle-related diseases, hypertension or body mass index addressing aim 2 and factors such as cardiorespiratory fitness addressing aim 3 will be considered. In case interaction terms are associated with the outcomes in a statistically significant manner $(\mathrm{p}<0.05)$, models will be stratified by that particular effect modifier.

To address research aim 4, the mediating effect of occupational and leisure-time physical activity on the association of socioeconomic status and the primary outcome, the product-of-coefficients test will be conducted, as described by MacKinnon. ${ }^{37}$ First, the association of socioeconomic status and the outcome (path c') will be estimated. Second, the association of socioeconomic status with the mediator (path b) will be estimated. Finally, the association between the mediator and outcome (path a) will be estimated. The product of coefficients $a \times b$ will provide an estimate of the relative strength of the mediation effect, while the proportion of mediation will be estimated by dividing the mediation effect $(\mathrm{a} \times \mathrm{b})$ by the total direct effect $\left(c^{\prime}=c^{\prime}+a \times b\right)$.

In all above-mentioned models (for aims 1-4), relevant confounders (eg, demographic, behavioural, psychosocial and material factors as shown in table 1 and figure 1) will be considered using a selection procedure in which all potential confounders will be univariately tested, after which a backward selection procedure will be conducted among variables with a statistically significant $(\mathrm{p}<0.10)$ univariate association with the primary outcome measure. Potential confounding variables with a multivariate statistically significant $(p<0.05)$ association with the primary outcome, will be retained in the final models.

\section{Assessment of meta-bias}

In the proposed research, various potential sources of bias will be considered. Reverse causality (ie, differences in health outcomes causing changes in physical activity, rather than the other way around) and the healthy worker effect (ie, relatively healthy workers being able to remain working in the most physically demanding jobs) will be assessed by performing a sensitivity analysis, excluding participants who have died within the first 5 years of the follow-up period. Bias due to differential misclassification (ie, physical activity measurement errors differing across groups) will be dealt with by conducting subgroup analyses on various factors (eg, subgroups with pre-existing cardiovascular conditions). Reporting bias of our work will be addressed by publishing the current protocol before merging and analysing the data. Moreover, reporting bias of original studies will be addressed by also including unpublished studies in our meta-analysis.

\section{Project and data management}

The active worker consortium core group (PC, MAH, WVM and AJvdB) at the Department of Public and Occupational Health, Amsterdam Public Health research institute, VU University Medical Centre will bear responsibility for the project coordination and scientific quality of the project. The core research group will be supplemented by an advisory group of international collaborators (AH, RT, 
PJM, SK and EC) within the active worker consortium, who will provide their knowledge, as well as their research data.

The core group, in close collaboration with the advisory group, will address the aforementioned research aims. Collaborating investigators are welcome to propose additional research projects. The advisory group, as well as any other collaborators providing data, will be asked to coauthor any future publication emerging from the consortium (with a maximum of two coauthors per participating study-with exceptions on reasonable request). However, to do so all coauthors will need to comply with the Recommendations for the Conduct, Reporting, Editing and Publication of Scholarly Work in Medical Journals (also known as the Vancouver Protocol for coauthorship). ${ }^{38}$ Collaborators are free to decline participation on a studyby-study basis, and have the right to withdraw their data from future analyses.

Data will be managed in accordance to The Netherlands Organisation for Health Research and Development (ZonMw) guidelines (https://www.zonmw.nl/en/ research-and-results/access-to-data), which includes the development of a data management plan stipulating the way in which the data will be safely store and made findable, accessible, interoperable and reusable.

The data inclusion procedure has started in May 2018 and we intend to continue doing so until the end of September 2018. As of 20 July 2018, researchers of a total of nine studies have already agreed to be part of the consortium, with two of them having transferred their data already. Of the other studies, application procedures for obtaining the data have started or studies were excluded from our consortium. Reasons for exclusion were the inability to get in contact with researchers (in some cases researchers have retired or have passed away) or the researchers not willing or being able to participate. Merging of the datasets and analysis syntaxes will be prepared in the period July to September 2018, to ensure that analyses can start when all data are collected in September 2018. Reporting on the data (in accordance to below-mentioned dissemination plan) is expected to be done by Mid-2019.

\section{Ethics and dissemination}

Outcomes of the active worker study will be published in at least two articles in scientific peer-reviewed journals (one on research aim 1 and one on research aims 2-4). If time permits, above-mentioned research aims will additionally be addressed using the secondary outcome measures.

\section{Patient and public involvement}

We currently describe a study protocol of a meta-analysis, hence no patient data have or will be collected. At this stage, there has not been any patient or public involvement.

\section{DISCUSSION}

This manuscript describes the rationale and design of the active worker consortium, an IPD meta-analysis. The primary aim of the active worker consortium is to explore the contribution of occupational and leisure-time physical activity to socioeconomic health differences, with the ultimate goal to improve the health of workers in lower socioeconomic status jobs.

Results from the proposed research will provide information on the potentially opposing health effects of occupational and leisure-time physical activity and the role they play in socioeconomic differences related to cardiovascular and all-cause mortality. Such information may help to clarify what lifestyle interventions may work for whom and when. This knowledge can guide interventions to improve the health of workers in certain lower socioeconomic status jobs.

As outlined in its recently drafted 'global action plan', ${ }^{39}$ WHO emphasises the importance of physical activity for a healthy world population. It has been estimated that physical inactivity is the fourth leading causes of lifestyle-related mortality as it is responsible for $>5$ million deaths globally each year. ${ }^{8}$ Moreover, physical inactivity has been estimated to account for $\sim 7 \%$ of the global health burden ${ }^{40}$ and is accompanied by considerable economic costs for our society. ${ }^{41}$ As such, physical activity is widely considered to be important for the prevention of several lifestyle-related diseases. ${ }^{8}$ The ultimate goal of our research is to address physical activity of workers in lower socioeconomic status jobs who are highly active at work, but not during their leisure time. It is likely that these workers may face the negative health effects of high dose of occupational physical activity and may only benefit to a limited extent from the positive health effects of leisuretime physical activity, simply because they are relatively physically inactive during leisure time.

A putative causal model for the physical activity paradox explaining this phenomenon is depicted in figure 3 . This figure, which is modified from earlier work ${ }^{19}$ and is theorised from existing evidence, shows the differential health consequences of occupational physical activity (being more common among those from lower socioeconomic status groups) and leisure-time physical activity (being more common among those from higher socioeconomic status groups). In this model it is hypothesised ${ }^{19}$ that high doses of occupational physical activity are often reached by occupational tasks, such as prolonged static postures and repetitive work (including manual handling and lifting), that are performed over long periods of time (eg, 40hours/week, during a lifelong work employment), with insufficient time for recovery. On the contrary, leisure-time physical activity typically takes place at high(er) intensity, often consisting of aerobic type of activities, that are only performed for a short time period, thereby allowing for recovery. Because of these natural differences, occupational and leisure-time physical activity likely differ in acute and chronic physiological responses. For example, high doses of occupational physical activity may not reach intensity levels required to achieve cardiorespiratory fitness improvements, ${ }^{42}$ but may cause chronic exhaustion and elevated resting blood 


\section{Nature \\ Acute \\ response \\ Chronic \\ response \\ Outcome}
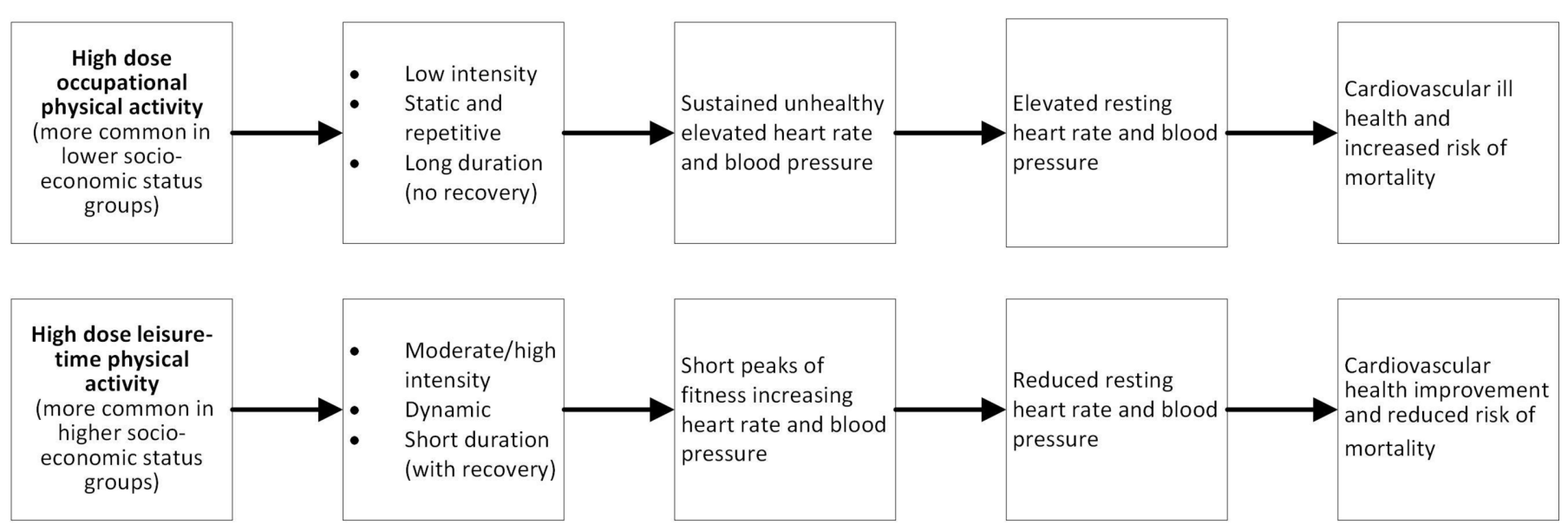

Figure 3 Putative causal model of the physical activity paradox showing the different health consequences of occupational and leisure-time physical activity on disease outcome. It is hypothesised that the nature of these two domains of physical activity differs considerably, resulting in different acute and chronic physiological responses and hence, health outcomes. Also, different mental and social health implications of work and leisure-time physical activities may play a role in this. For example, leisure-time physical activity is often associated with positive outcomes of mental health and social functioning, while highly demanding jobs often come with mental strain which has been shown to be associated with negative health outcomes.

pressure $^{43}$ and elevated resting heart rate ${ }^{44}$ that may ultimately result in cardiovascular diseases and mortality. 4546 On the other hand, leisure-time physical activity may be cardiorespiratory fitness enhancing, ${ }^{47}$ and reduce resting blood pressure and heart rate, thereby reducing the risk of cardiovascular disease and mortality. Also, different mental and social health implications of work and leisure-time physical activities may play a role in this. For example, leisure-time physical activity is often associated with positive outcomes of mental health (such as mood states like anxiety, stress and depression $)^{48}$ and social functioning (such as loneliness and social support). ${ }^{49}$ On the other hand, highly demanding jobs often come with mental strain which has been shown to be associated with negative health outcomes, including cardiovascular disorders. ${ }^{50}$

According to this hypothesis, it may be of importance to encourage workers from physically demanding occupations to be also physically active during leisure time. As this message may be considered counterintuitive, a strong body of evidence is required. The current study will further strengthen the evidence base regarding the health implications of occupational and leisure-time physical activity. However, mechanisms underlying this association, such as the ones shown in figure 3 , should also be investigated in studies with other research methodologies, for example, in field measurements using ambulatory measurements of physical activity, biomechanical load, heart rate and blood pressure.

Besides, any public health policy or intervention to promote a physically active lifestyle will, however, be confronted with the dilemma that such initiatives usually do not reach those who would benefit the most, that is, workers with a low socioeconomic status. ${ }^{51}$ Targeted interventions for these workers are therefore needed, for which the results of the proposed research will be of importance.

As far as we are aware there is currently no data available on objectively measured physical activity for which: (1) work chorus can be distinguished from leisure-time chorus and (2) physical activity can be linked to mortality outcome data. A limitation of the current study will therefore be that all data will be based on self-reports, which may be biased.$^{52}$

\section{Author affiliations}

${ }^{1}$ Department of Public and Occupational Health, Amsterdam Public Health Research Institute, Amsterdam UMC, Vrije Universiteit Amsterdam, Amsterdam, The Netherlands

${ }^{2}$ National Research Centre for the Working Environment, Copenhagen, Denmark ${ }^{3}$ Risk Factor Assessment Branch, Division of Cancer Control and Population Sciences, US National Cancer Institute, Rockville, Maryland, USA

${ }^{4}$ Department of Public Health and Nursing, Norwegian University of Science and Technology, Trondheim, Norway

${ }^{5}$ Department of Public Health, Ghent University, Ghent, Belgium

Contributors PC has drafted the current manuscript. All authors (PC, MAH, AH, RT, PJM, SK, EC, WVM and AJvdB) were involved in the design of this study, acquisition of research funding for this project and reviewed the manuscript for important intellectual content. AJvdB is the study guarantor.

Funding This study has been funded by The Netherlands Organisation for Health Research and Development; ZonMw (grant \#: 531-00141-3).

Disclaimer The funding body played no role in the development of this protocol. Competing interests None declared.

Patient consent Not required. 
Ethics approval According to Dutch legislation, the Medical Ethical Committee of the VU University Medical Center has declared that the Medical Research Involving Human Subjects Act does not apply to the current study. As such no ethics approval is required for the current study.

Provenance and peer review Not commissioned; externally peer reviewed.

Open access This is an open access article distributed in accordance with the Creative Commons Attribution Non Commercial (CC BY-NC 4.0) license, which permits others to distribute, remix, adapt, build upon this work non-commercially, and license their derivative works on different terms, provided the original work is properly cited, appropriate credit is given, any changes made indicated, and the use is non-commercial. See: http://creativecommons.org/licenses/by-nc/4.0/.

\section{REFERENCES}

1. Mackenbach JP, Stirbu I, Roskam AJ, et al. Socioeconomic inequalities in health in 22 European countries. $N$ Engl J Med 2008;358:2468-81.

2. Kunst AE, Bos V, Lahelma E, et al. Trends in socioeconomic inequalities in self-assessed health in 10 European countries. Int $J$ Epidemiol 2005;34:295-305.

3. Granström F, Molarius A, Garvin P, et al. Exploring trends in and determinants of educational inequalities in self-rated health. Scand $J$ Public Health 2015;43:677-86.

4. van Lenthe FJ, Schrijvers CT, Droomers M, et al. Investigating explanations of socio-economic inequalities in health: the Dutch GLOBE study. Eur J Public Health 2004;14:63-70.

5. Laaksonen M, Roos E, Rahkonen O, et al. Influence of material and behavioural factors on occupational class differences in health. $J$ Epidemiol Community Health 2005;59:163-9.

6. Kivimäki M, Gimeno D, Ferrie JE, et al. Socioeconomic position, psychosocial work environment and cerebrovascular disease among women: the Finnish public sector study. Int J Epidemiol 2009;38:1265-71.

7. Moor I, Spallek J, Richter M. Explaining socioeconomic inequalities in self-rated health: a systematic review of the relative contribution of material, psychosocial and behavioural factors. J Epidemiol Community Health 2017;71:565-75.

8. Lee IM, Shiroma EJ, Lobelo F, et al. Effect of physical inactivity on major non-communicable diseases worldwide: an analysis of burden of disease and life expectancy. Lancet 2012;380:219-29.

9. Sallis JF, Owen N, Fisher EB. Ecological models of health behavior. In: Glanz K, Rimer BK, Viswanath K, eds. Health behavior and health education: theory, research, and practice. 4th ed.. San Francisco, CA: Jossey-Bass, 2008:465-82.

10. Li J, Loerbroks A, Angerer P. Physical activity and risk of cardiovascular disease: what does the new epidemiological evidence show? Curr Opin Cardiol 2013;28:575-83.

11. Holtermann A, Hansen JV, Burr H, et al. The health paradox of occupational and leisure-time physical activity. Br J Sports Med 2012;46:291-5.

12. Holtermann A, Marott JL, Gyntelberg F, et al. Occupational and leisure time physical activity: risk of all-cause mortality and myocardial infarction in the Copenhagen City Heart Study. A prospective cohort study. BMJ Open 2012;2:e000556.

13. Petersen CB, Eriksen L, Tolstrup JS, et al. Occupational heavy lifting and risk of ischemic heart disease and all-cause mortality. BMC Public Health 2012;12:1070.

14. Hu GC, Chien KL, Hsieh SF, et al. Occupational versus leisure-time physical activity in reducing cardiovascular risks and mortality among ethnic Chinese adults in Taiwan. Asia Pac J Public Health 2014;26:604-13

15. Krause N, Brand RJ, Arah OA, et al. Occupational physical activity and 20-year incidence of acute myocardial infarction: results from the Kuopio Ischemic Heart Disease Risk Factor Study. Scand J Work Environ Health 2015;41:124-39.

16. Clays E, Lidegaard M, De Bacquer D, et al. The combined relationship of occupational and leisure-time physical activity with all-cause mortality among men, accounting for physical fitness. Am J Epidemiol 2014;179:559-66.

17. Harari G, Green MS, Zelber-Sagi S. Combined association of occupational and leisure-time physical activity with all-cause and coronary heart disease mortality among a cohort of men followed-up for 22 years. Occup Environ Med 2015;72:617-24.

18. Richard A, Martin B, Wanner M, et al. Effects of leisure-time and occupational physical activity on total mortality risk in NHANES III according to sex, ethnicity, central obesity, and age. J Phys Act Health 2015;12:184-92.
19. Holtermann A, Krause N, van der Beek AJ, et al. The physical activity paradox: six reasons why occupational physical activity (OPA) does not confer the cardiovascular health benefits that leisure time physical activity does. Br J Sports Med 2018;52.

20. Coenen P, Huysmans MA, Holtermann A, et al. Do highly physically active workers die early? A systematic review with meta-analysis of data from 193696 participants. Br J Sports Med 2018;52:1320-6.

21. van Dommelen P, Coffeng JK, van der Ploeg HP, et al. Objectively measured total and occupational sedentary time in three work settings. PLoS One 2016;11:e0149951.

22. Beenackers MA, Kamphuis CB, Giskes K, et al. Socioeconomic inequalities in occupational, leisure-time, and transport related physical activity among European adults: a systematic review. Int $J$ Behav Nutr Phys Act 2012;9:116.

23. Pampel FC, Krueger PM, Denney JT. Socioeconomic disparities in health behaviors. Annu Rev Sociol 2010;36:349-70.

24. Berlin JA, Santanna J, Schmid CH, et al. Individual patient- versus group-level data meta-regressions for the investigation of treatment effect modifiers: ecological bias rears its ugly head. Stat Med 2002;21:371-87

25. Cochrane Individual Participant Data (IPD). Meta-analysis methods group. http://ipdmamg.cochrane.org/

26. Riley RD, Lambert PC, Abo-Zaid G. Meta-analysis of individual participant data: rationale, conduct, and reporting. $B M J$ 2010;340:c221.

27. Shamseer L, Moher D, Clarke M, et al. Preferred reporting items for systematic review and meta-analysis protocols (PRISMA-P) 2015: elaboration and explanation. BMJ 2015;350:g7647.

28. Coenen P, Huysmans MA, Holtermann A, et al. Can socio-economic health differences be explained by physical activity? The active worker consortium; an individual participant meta-analysis. Prospero 2018:CRD42018085228.

29. Stewart LA, Clarke M, Rovers M, et al. Preferred Reporting Items for Systematic Review and Meta-Analyses of individual participant data: the PRISMA-IPD Statement. JAMA 2015;313:1657-65.

30. Buffart LM, Kalter J, Chinapaw MJ, et al. Predicting OptimaL cAncer Rehabllitation and Supportive care (POLARIS): rationale and design for meta-analyses of individual patient data of randomized controlled trials that evaluate the effect of physical activity and psychosocial interventions on health-related quality of life in cancer survivors. Syst Rev 2013;2:75

31. Buffart LM, Kalter J, Sweegers MG, et al. Effects and moderators of exercise on quality of life and physical function in patients with cancer: An individual patient data meta-analysis of $34 \mathrm{RCTs}$. Cancer Treat Rev 2017;52:91-104.

32. IPAQ, 2005. Guidelines for data processing and analysis of the international physical activity questionnaire (IPAQ) - Short and long forms https://sites.google.com/site/theipaq/.

33. Tudor-Locke C, Ainsworth BE, Washington TL, et al. Assigning metabolic equivalent values to the 2002 census occupational classification system. Journal of Physical Activity and Health 2011;8:581-6.

34. Ainsworth BE, Haskell WL, Whitt MC, et al. Compendium of physical activities: an update of activity codes and MET intensities. Med Sci Sports Exerc 2000;32:S498-S516.

35. Tremblay MS, Colley RC, Saunders TJ, et al. Physiological and health implications of a sedentary lifestyle. Appl Physiol Nutr Metab 2010;35:725-40.

36. Hayden JA, van der Windt DA, Cartwright JL, et al. Assessing bias in studies of prognostic factors. Ann Intern Med 2013;158:280-6.

37. MacKinnon DP. Introduction to Statistical Mediation Analysis. Abingdon, UK: Taylor \& Francis Inc, 2008.

38. International Committee of Medical Journal Editors. Recommendations for the Conduct, Reporting, Editing, and Publication of Scholarly Work in Medical Journals, 2017.

39. World Health Organization. Draft WHO global action plan on physical activity 2018 - 2030. Geneva, Switzerland: World Health Organization, 2017.

40. Begg SJ, Vos T, Barker B, et al. Burden of disease and injury in Australia in the new millennium: measuring health loss from diseases, injuries and risk factors. Med J Aust 2008;188:36-40.

41. Ding D, Lawson KD, Kolbe-Alexander TL, et al. The economic burden of physical inactivity: a global analysis of major non-communicable diseases. Lancet 2016;388:1311-24.

42. Korshøj M, Krustrup P, Jespersen T, et al. A 24-h assessment of physical activity and cardio-respiratory fitness among female hospital cleaners: a pilot study. Ergonomics 2013;56:935-43.

43. Clays E, De Bacquer D, Van Herck K, et al. Occupational and leisure time physical activity in contrasting relation to ambulatory blood pressure. BMC Public Health 2012;12:1002. 
44. Jakobsen MD, Sundstrup E, Persson R, et al. Is Borg's perceived exertion scale a useful indicator of muscular and cardiovascular load in blue-collar workers with lifting tasks? A cross-sectional workplace study. Eur J Appl Physiol 2014;114:425-34.

45. Palatini P. Elevated heart rate: a "new" cardiovascular risk factor? Prog Cardiovasc Dis 2009;52:1-5.

46. Xie X, Atkins E, Lv J, et al. Effects of intensive blood pressure lowering on cardiovascular and renal outcomes: updated systematic review and meta-analysis. Lancet 2016;387:435-43.

47. Eriksen L, Grønbaek M, Helge JW, et al. Cardiorespiratory fitness in 16025 adults aged $18-91$ years and associations with physical activity and sitting time. Scand J Med Sci Sports 2016;26:1435-43.

48. Mikkelsen K, Stojanovska L, Polenakovic M, et al. Exercise and mental health. Maturitas 2017;106:48-56.

49. Shvedko A, Whittaker AC, Thompson JL, et al. Physical activity interventions for treatment of social isolation, loneliness or low social support in older adults: A systematic review and metaanalysis of randomised controlled trials. Psychol Sport Exerc 2018;34:128-37.

50. Nyberg ST, Fransson El, Heikkilä K, et al. Job strain and cardiovascular disease risk factors: meta-analysis of individualparticipant data from 47,000 men and women. PLoS One 2013;8:e67323.

51. Wardle J, Steptoe A. Socioeconomic differences in attitudes and beliefs about healthy lifestyles. $J$ Epidemiol Community Health 2003;57:440-3.

52. Gupta N, Heiden M, Mathiassen SE, et al. Is self-reported time spent sedentary and in physical activity differentially biased by age, gender, body mass index, and low-back pain? Scand J Work Environ Health 2018;44:163-70. 